\title{
Simultaneous occurrence of polar stratospheric clouds and upper-tropospheric clouds caused by blocking anticyclones in the Southern Hemisphere
}

\author{
M. Kohma and K. Sato \\ Department of Earth and Planetary Science, The University of Tokyo, Tokyo, Japan \\ Correspondence to: M. Kohma (kohmasa@eps.s.u-tokyo.ac.jp) \\ Received: 12 June 2012 - Published in Atmos. Chem. Phys. Discuss.: 10 August 2012 \\ Revised: 8 March 2013 - Accepted: 21 March 2013 - Published: 12 April 2013
}

\begin{abstract}
Previous studies have reported that polar stratospheric clouds (PSCs) are frequently observed simultaneously with upper-tropospheric clouds (UCs) in the Southern Hemisphere. However, it has not yet been examined whether the UCs that simultaneously occur with PSCs are actually located below the height of the tropopause, which is modified by tropospheric disturbances. Furthermore, the mechanism of this simultaneous occurrence has not yet been clarified. This study statistically examines the simultaneous appearance of PSCs and UCs using the Cloud-Aerosol Lidar and Pathfinder Satellite Observation (CALIPSO) for the five austral winters of 2007-2011. From correlation analyses and statistical dependence tests, it is shown that the simultaneous occurrence frequencies of clouds with an altitude range of $15-25 \mathrm{~km}$ and $9-11 \mathrm{~km}$ are significant. The analyses based on tropopause-relative altitude suggest that the occurrence frequency of clouds at altitudes higher than $6 \mathrm{~km}$ above the local tropopause (i.e., PSCs) is significantly correlated with that of clouds around and slightly above the tropopause. These results indicate that the UCs observed simultaneously with PSCs reported in previous case studies are likely located around and slightly above the tropopause rather than in the troposphere. It is also shown that the simultaneous occurrence of PSCs and UCs is frequently associated with blocking highs that have large horizontal scales (several thousand kilometers) and tall structure (up to a height of $\sim 15 \mathrm{~km}$ ). The longitudinal variation of blocking high frequency accords well with that of the simultaneous occurrence frequency of PSCs and UCs. This fact suggests that the blocking highs provide a preferable condition for such simultaneous occurrences. Moreover, the composition of PSCs is investigated as
\end{abstract}

a function of relative longitude of the anticyclones including blocking highs. It was discovered that relatively high proportions of STS (super-cooled ternary solutions), Ice, and Mix2 (mixture of nitric acid trihydrate and STS) types are distributed towards the windward, near, and leeward side of anticyclones in westerly background flows, respectively.

\section{Introduction}

Polar stratospheric clouds (PSCs), which appear in the polar stratospheric winter, play a significant role in the depletion of polar stratospheric ozone. They provide an environment for the heterogeneous reactions that convert stable chlorine and bromine reservoirs to active forms, and they lead to the irreversible removal of $\mathrm{NO}_{\mathrm{y}}$ from the lower stratosphere (denitrification) (Solomon, 1999). From lidar observations, it is shown that PSCs are mainly composed of nitric acid trihydrate (Type 1a, NAT), super-cooled ternary solution (Type $1 \mathrm{~b}$, STS), and/or ice particles (Type 2) (Lowe and MacKenzie, 2008). Large NAT particles are considered to be responsible for efficient denitrification due to their rapid gravitational sedimentation (Jensen et al., 2002; Fueglistaler et al., 2002; Lambert et al., 2012).

PSCs are strongly modulated by atmospheric waves such as planetary, synoptic-scale and gravity waves (Teitelbaum et al., 2001; Carslaw et al., 1999; Dörnbrack and Leutbecher, 2001; Eckermann et al., 2009; McDonald et al., 2009; Alexander et al., 2011; Kohma and Sato, 2011). Teitelbaum et al. (2001) showed that PSCs and synoptic-scale ozone minima appear simultaneously with synoptic-scale 
anticyclones around the tropopause in the Northern Hemisphere. They concluded that anticyclones near the tropopause have a primary role in the occurrence of Arctic PSCs. Fromm et al. (2003), who used long-term satellite observations that included the Stratospheric Aerosol Measurement II, Stratospheric Aerosol and Gas Measurement II, and Polar Ozone and Aerosol Measurement II/III, also confirmed that Arctic PSCs are frequently associated with an elevated tropopause $(\sim 10 \mathrm{~km})$, which is attributable to anticyclones near the tropopause (Wirth, 2000). Anticyclonic potential vorticity (PV) anomalies near the tropopause are associated with low temperatures above the tropopause (e.g., Holton, 2004): a PV anomaly at one level yields nonzero geopotential anomalies at levels above and below this level. The amplitude of a geopotential anomaly has a maximum at the level of the PV anomaly. As a temperature anomaly is proportional to the vertical derivative of a geopotential anomaly, an anticyclonic PV anomaly at one level is accompanied with low (high) temperatures above (below) it.

McDonald et al. (2009), who used Challenging MiniSatellite Payload (CHAMP) radio occultation observations, showed that gravity waves increase the frequency of temperatures below the NAT equilibrium temperature in June in the Antarctic, especially around the Antarctic Peninsula, which is a mountain wave "hotspot". Dörnbrank and Leutbecher (2001) showed that temperatures low enough to enable the formation of ice PSCs are controlled by mesoscale temperature fluctuations due to mountain waves in the Arctic, while NAT formation is significantly controlled by the synopticscale temperature fluctuations. Kohma and Sato (2011) quantified the contribution of each type of waves to the areal extent of PSCs in both hemispheres using a temperature threshold based on temperatures from reanalysis data and GPS radio occultation observations and mixing ratios of $\mathrm{HNO}_{3}$ and $\mathrm{H}_{2} \mathrm{O}$ from microwave limb sounder observations. They showed that planetary-scale temperature perturbations have a great contribution to areal extent of PSCs in the latitude range of $55-70^{\circ} \mathrm{S}$ while the contribution of synoptic-scale perturbation is large only around an altitude of $12 \mathrm{~km}$. The composition of PSCs is also affected by atmospheric waves, particularly by gravity waves (e.g., Carslaw et al., 1998; Höpfner et al., 2006; Eckermann et al., 2009). Höpfner et al. (2006) examined a sudden occurrence of circumpolar NAT distributions in June 2003 and attributed it to the heterogeneous nucleation on ice particles formed in low-temperature perturbations associated with mountain waves over the Antarctic Peninsula. From COSMIC/FORMOSAT observations in the Southern Hemisphere, Alexander et al. (2011) showed that ice and NAT PSCs are more frequent when and where the variances of temperature fluctuations associated with gravity waves are large.

Recent studies that used satellite observations (Palm et al., 2005; Wang et al., 2008; Adhikari et al., 2010) have reported that PSCs in the Antarctic are frequently observed simultaneously with upper-tropospheric clouds (UCs). Palm et al. (2005) suggested that tropospheric disturbances may contribute to the formation of PSCs. Adhikari et al. (2010) examined PSC compositions and showed that the fraction of ice PSCs increases in association with high and deep tropospheric clouds. Recently, Achtert et al. (2012) showed that $72 \%$ of the Arctic PSCs observed by the Cloud-Aerosol Lidar and Infrared Pathfinder Satellite Observation (CALIPSO) in the winter of 2007/2008 were accompanied by deep tropospheric cloud systems. However, the mechanisms causing the simultaneous occurrence of PSCs and UCs are still unclear. In addition, locations of the UCs that are simultaneously observed with PSCs are close to the tropopause $(8-9 \mathrm{~km}$ in the polar winter). For example, Wang et al. (2008) only considered clouds with a top and bottom below an altitude of $15 \mathrm{~km}$ and $12 \mathrm{~km}$, respectively, as tropospheric clouds. However, tropopause height varies in time and space. Thus, it should be ascertained whether UCs that occur simultaneously with PSCs are actually tropospheric clouds.

This study statistically examines the frequency of simultaneous observations of PSCs and UCs using satellite lidar observation from the Cloud-Aerosol Lidar with Orthogonal Polarization (CALIOP) and reanalysis data from the ECMWF re-analysis (ERA)-Interim of the five austral winters of 2007-2011. A description of the data used in the present study is provided in Sect. 2. In Sect. 3, results are discussed in the following manner: first, statistical analyses of the simultaneous occurrence of PSCs and UCs are made. Second, composite analyses and a detailed case study are made to show that the tall structure of blocking highs is an important factor for the simultaneous occurrence of PSCs and UCs. Third, after considering an appropriate definition of blocking highs, longitudinal distribution similarities of the occurrence frequency of blocking highs and of the simultaneous occurrence of PSCs and UCs are examined. Finally, the fraction of each PSC composition is shown as a function of longitude relative to anticyclones. In Sect. 4, summary and concluding remarks are given.

\section{Data description}

\subsection{CALIOP data}

In the present analysis, the CALIPSO lidar Level 1B Profile data product (version 3) is used to examine PSCs and UCs. This data product includes profiles of total attenuated backscatter and perpendicular attenuated backscatter at $532 \mathrm{~nm}$ at an altitude range of up to $40 \mathrm{~km}$ and covers a latitudinal region of $82^{\circ} \mathrm{S}-82^{\circ} \mathrm{N}$. An algorithm proposed by Pitts et al. (2009) is used to detect PSCs. In this algorithm, not only the scattering ratios but also the depolarization ratios of aerosols are used for the detection of optically thin clouds (low number densities/volumes). Pitts et al. (2009) found that the inclusion of depolarization ratio results in an increase of the areal coverage of PSCs by $15 \%$ when compared to an 
algorithm only using scattering ratios. Before applying their algorithm, the original data are averaged every $5 \mathrm{~km}$ horizontally and every $180 \mathrm{~m}$ vertically. Moreover, following Pitts et al. (2009), only nighttime data are analyzed for regions between $55-82^{\circ} \mathrm{S}$, where most PSCs are observed. As it is not clear whether UCs are clouds that actually occur in the troposphere, the occurrence frequency of clouds is defined as the percentage of fractions where any cloud (PSC or UC) was observed. The occurrence frequency of these clouds (hereinafter referred to as PSC/UC frequency) is calculated for each day in each vertical $1 \mathrm{~km}$ bin in regions with longitude intervals of $10^{\circ}$ for five latitude bands: $55-60^{\circ} \mathrm{S}, 60-65^{\circ} \mathrm{S}$, $65-70^{\circ} \mathrm{S}, 70-75^{\circ} \mathrm{S}$ and $75-82^{\circ} \mathrm{S}$. The total number of observations is approximately 500 in June and 350 in September for each grid box of $70-75^{\circ} \mathrm{S}$.

While the algorithm was originally intended to categorize PSCs in 400-700 K potential temperature levels, Alexander et al. (2010) suggested that the same PSC detection algorithm can be applied to regions extending down to the $350 \mathrm{~K}$ level, based on the fact that the PSC distributions of the 350-400 K levels are similar to those of the upper layers. To examine PSCs in the lowermost stratosphere and UCs without specific distinctions, the present study further applies the same algorithm down to the regions at $300 \mathrm{~K}$. Thus, any cloud could be analyzed in regions with potential temperatures from 300 to $700 \mathrm{~K}(8-28 \mathrm{~km}$ in polar latitudes) and are referred to as PSC/UC. PSC/UC frequency is calculated in the altitude range of $9-30 \mathrm{~km}$ at intervals of $1 \mathrm{~km}$.

The CALIPSO lidar Level 2 Vertical Feature Mask (VFM) data are used to analyze the clouds below $9 \mathrm{~km}$. The VFM data product categorizes each segment into a feature type: "clear air", "cloud", and "stratospheric". When calculating PSC/UC frequency, both "cloud", and "stratospheric" feature types are used to determine cloud existence in the present study. Similar to the PSC/UC frequencies of the CALIPSO lidar Level 1B data, PSC/UC frequency is calculated in the altitude range of 3-9 km using the Level 2 VFM data. Because the cloud and aerosol detection algorithm of the Level 2 data is based on the total attenuated backscatter coefficients (or scattering ratios), it may overlook the optically thin stratospheric clouds. Thus, the Level 1B data are used for the altitude range of 9-30 km, where PSCs are often observed.

To ensure that the algorithm for the Level 1B data works in the lowermost stratosphere and upper troposphere, a Hovmöller diagram of PSC/UC frequency at altitudes of 9$11 \mathrm{~km}$ was made for June through September 2008 (Fig. 1a). For comparison, a plot of PSC/UC frequency made from the CALIPSO lidar Level 2 VFM data is also shown (Fig. 1b). The distributions observed in these two plots are quite similar, indicating that the algorithm by Pitts et al. (2009) can be used to detect clouds in regions as low as the $300 \mathrm{~K}$ level correctly in terms of occurrence frequency.

In addition, based on inverse scattering ratios and depolarization ratios, PSCs are categorized into four groups: Ice, STS (super-cooled ternary solution), Mix 1 (mixture with
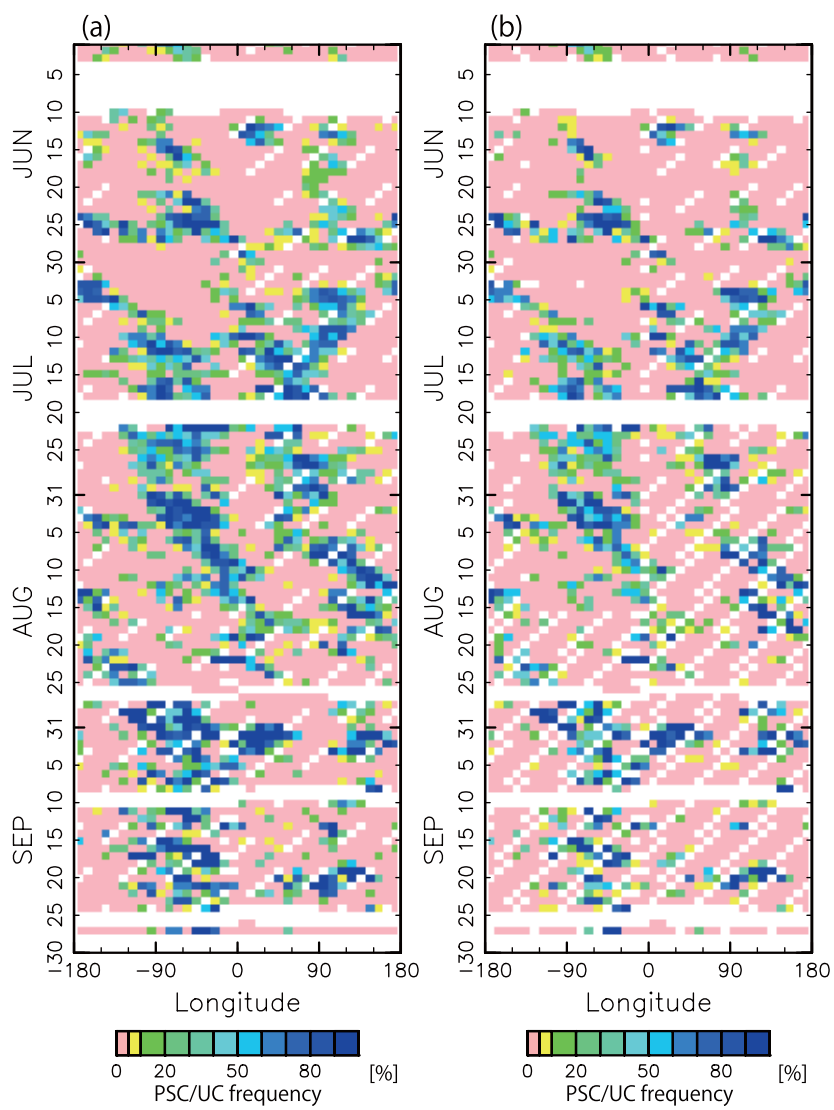

Fig. 1. Hovmöller diagrams of PSC/UC frequency from (a) the CALIPSO lidar Level 1B data and (b) CALIPSO lidar Level 2 VFM data, both averaged within an altitude range of $9-11 \mathrm{~km}$ in the latitude range of $70-75^{\circ} \mathrm{S}$ for the austral winter of 2008 .

a lower number/volume of NAT particles and STS particles) and Mix2 (mixture with a higher number/volume of NAT particles and STS particles) (Pitts et al., 2009). Pitts et al. (2009) categorized PSC composition into twodimensional space of the inverse scattering ratio versus the depolarization ratio. The PSC composition below $15 \mathrm{~km}$ $(\sim 400 \mathrm{~K})$, which is the upper limit of the tropopause height, is not examined in the present study due to possible contaminations from tropospheric clouds and aerosols.

\subsection{Tropopause height definitions}

To examine PSC/UC heights relative to the tropopause, a local tropopause height is calculated for each grid and day using daily averaged ERA-Interim data. The ERA-Interim data are distributed in $1.5^{\circ} \times 1.5^{\circ}$ grids and at 37 levels between 1 and $1000 \mathrm{hPa}$. The tropopause height is usually determined using temperature lapse rates (thermal tropopause; WMO, 1957). However, the definition of the thermal tropopause frequently gives inappropriate estimations for polar night region as the static stability is quite low in the lower stratosphere there. It is well known that discontinuity of 
tropopause heights and multivalued tropopause heights are seen (e.g., Wilcox et al., 2012). Thus, the definition of the tropopause that was proposed by Wilcox et al. (2012) was used. This tropopause comprises both thermal and dynamical tropopause; it is defined as

$h_{\mathrm{b}}=\left\{\begin{array}{l}h_{\mathrm{d}} \text { for } \theta_{\mathrm{d}}<350 \mathrm{~K}, \\ \left(\frac{\theta_{\mathrm{d}}-350}{20}\right) h_{\mathrm{m}}+\left(1-\frac{\theta_{\mathrm{d}}-350}{20}\right) h_{\mathrm{d}} \text { for } 350 \mathrm{~K}<\theta_{\mathrm{d}}<370 \mathrm{~K}, \\ h_{\mathrm{m}} \text { for } \theta_{\mathrm{d}}>370 \mathrm{~K},\end{array}\right.$

where $\theta_{\mathrm{d}}$ is the potential temperature at the dynamical tropopause and $h_{\mathrm{m}}$ is the lowest of either the thermal tropopause height $\left(h_{\mathrm{t}}\right)$ or dynamical tropopause height $\left(h_{\mathrm{d}}\right)$. Here, $h_{\mathrm{t}}$ is calculated following Reichler et al. (2003). The dynamical tropopause is defined as the height corresponding to $\mathrm{PV}=-2 \mathrm{PVU}\left(\mathrm{PVU}=10^{-6} \mathrm{~m}^{2} \mathrm{~s}^{-1} \mathrm{~K} \mathrm{~kg}^{-1}\right)$. Kunz et al. (2011) showed that this constant value of PV roughly corresponds to the PV gradient-based tropopause in high latitudes of the Southern Hemisphere during the entire year. The average and standard deviation of the blended tropopause $\left(h_{\mathrm{b}}\right)$ height in the latitude range of $55-82^{\circ} \mathrm{S}$ from June to September are 8.5 and $1.4 \mathrm{~km}$, respectively. When the tropopause heights are compared with PSC/UC frequency in the following analyses, the tropopause heights are linearly interpolated into the same horizontal grids as those of PSC/UC frequency.

Figure 2a shows the frequency distribution of $h_{\mathrm{b}}$ and $h_{\mathrm{t}}$ in the latitude range of $60-82^{\circ} \mathrm{S}$ from June to September 2007. The results show that $h_{\mathrm{t}}$ tend to be higher than $h_{\mathrm{b}}$. The difference between the two amounts to $7 \mathrm{~km}$, which is mainly due to anomalously high $h_{\mathrm{t}}$ values. A comparison between $h_{b}$ and $h_{\mathrm{d}}$ is shown in Fig. 2b. In the latitude region of $60-82^{\circ} \mathrm{S}, h_{\mathrm{b}}$ and $h_{\mathrm{d}}$ accord well.

It is noteworthy that the classification of "cloud" and "stratospheric" flags in the Level 2 data is based on the thermal tropopause defined using the Goddard Earth Observing System Model Version 5 (GEOS-5) meteorological data, and hence should not be reliable in the polar winter due to their issues with thermal tropopause heights (CALIPSO Quality Statement Summary (eosweb.larc.nasa.gov/PRODOCS/ calipso)).

To confirm this, the numbers of "cloud" and "stratospheric" flags are examined for $60-82^{\circ} \mathrm{S}$ in the altitude range of 8-20 km for respective months from June to September in 2007 as a function of the blended tropopause (Table 1). The tropopause is determined by linear interpolation at each horizontal location of the cloud profile. The number of "stratospheric" flags below $h_{\mathrm{b}}$ is quite small compared to the total number of "stratospheric" flags. However, $24 \%$ of "cloud" flags are located in $z>h_{\mathrm{b}}+2 \mathrm{~km}$. In other words, if "cloud" flags in $z>h_{\mathrm{b}}+2 \mathrm{~km}$ were treated as tropospheric clouds, the number of clouds in the stratosphere should significantly decrease. Thus, "cloud" and "stratospheric" flags should not be used to classify clouds as PSCs and tropospheric clouds.

Based on these facts, in the present study the daily $\mathrm{PSC} / \mathrm{UC}$ frequencies in the altitude range of $9-30 \mathrm{~km}$ and 3-

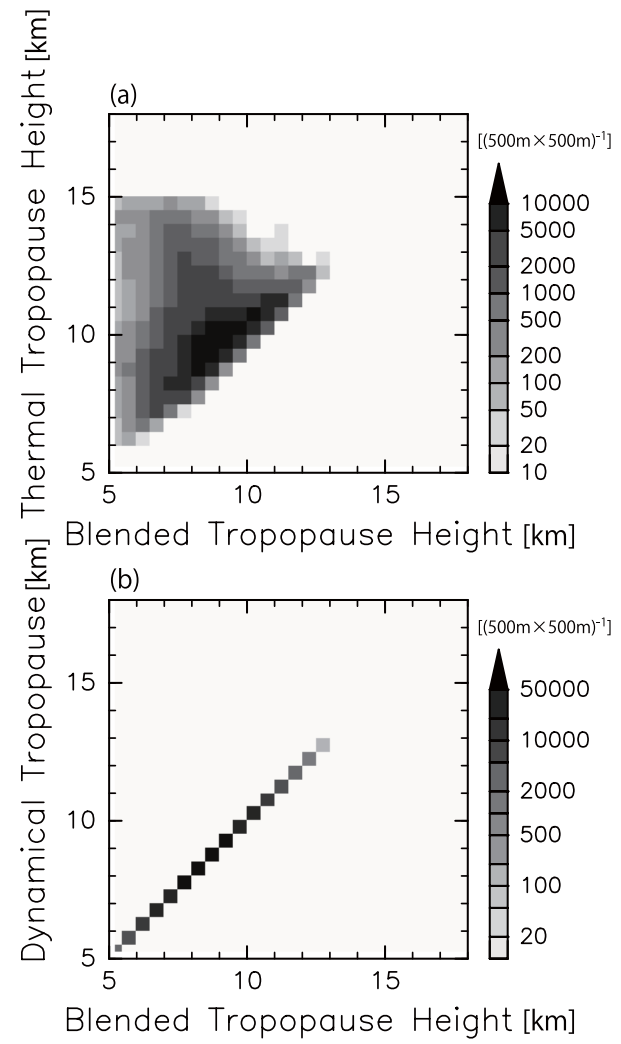

Fig. 2. (a) Two-dimensional frequency distribution of altitude for the blended and thermal tropopause in the latitude range of 60 $82^{\circ} \mathrm{S}$ from June through September 2007. (b) The same as (a) but for the frequency distribution of altitudes for the blended and the dynamical tropopause.

$9 \mathrm{~km}$ and at vertical intervals of $1 \mathrm{~km}$ are calculated from the CALIPSO lidar Level 1B data and Level 2 data, respectively. Local tropopause heights are calculated from the daily mean ERA-Interim data following the definition of Wilcox et al. (i.e., $h_{\mathrm{b}}$ ). For the analysis of atmospheric disturbances, daily mean ERA-Interim data are used.

\section{Results}

\subsection{The relation between PSCs and UCs}

The right panels of Fig. 3 show zonally averaged PSC/UC frequency as a function of altitude for $70-75^{\circ} \mathrm{S}$ and are obtained using data from June through September 2007-2011. It should be noted that the discontinuity around $20 \mathrm{~km}$ is due to the difference in the original vertical resolution of the CALIPSO lidar Level 1B data below and above this level and should be ignored.

A minimum can be seen in the vertical profiles of PSC/UC frequency at an altitude range of $12-14 \mathrm{~km}$ in June and July. This minimum gradually becomes obscured with time. This feature is most likely related to the seasonal variation of 
Table 1. Number of the "cloud" and "stratospheric" flags from the CALIPSO Level 2 VFM data in the latitude range of $60-82^{\circ} \mathrm{S}$ and in the altitude range of 8-20 km in 2007 when (a) $z<h_{\mathrm{b}}$, (b) $h_{\mathrm{b}}<z<h_{\mathrm{b}}+2 \mathrm{~km}$, and (c) $h_{b}+2 \mathrm{~km}<z$, where $z$ and $h_{\mathrm{b}}$ are the altitude and the tropopause height, respectively, following the definition of Wilcox et al. The percentages in parentheses indicate the percentage of each flag in the given altitude range.

\begin{tabular}{llrrr}
\hline & & (a) & (b) & (c) \\
& & $z<h_{\mathrm{b}}$ & $h_{\mathrm{b}}<z<h_{\mathrm{b}}+2 \mathrm{~km}$ & $h_{\mathrm{b}}+2 \mathrm{~km}<z$ \\
\hline June & "cloud" flag & 52385 & 52026 & 24930 \\
& "stratospheric" flag & 4062 & 17393 & 37736 \\
July & "cloud" flag & 52376 & 52129 & 39227 \\
& "stratospheric" flag & 2850 & 25771 & 44234 \\
August & "cloud" flag & 54236 & 54884 & 41815 \\
& "stratospheric" flag & 2560 & 26607 & 45837 \\
\multirow{3}{*}{ JJAS } & "cloud" flag & 48849 & 49333 & 27927 \\
& "stratospheric" flag & 1326 & 11519 & 21981 \\
& "cloud" flag & $207846(38 \%)$ & $208372(38 \%)$ & $133899(24 \%)$ \\
& "stratospheric" flag & $10798(5 \%)$ & $66202(29 \%)$ & $149788(66 \%)$ \\
\hline
\end{tabular}

altitudes where PSCs are observed. According to previous studies (Poole and Pitts, 1994; Adriani et al., 2004; Pitts et al., 2007), the altitudes where PSCs dominantly occur decrease from $22 \mathrm{~km}$ in June to $15 \mathrm{~km}$ or below in September and October following the lowest temperature descent and an irreversible loss of $\mathrm{HNO}_{3}$ and $\mathrm{H}_{2} \mathrm{O}$ due to the sedimentation of PSCs. In the following, the levels in which PSC/UC frequencies in the upper levels $(15-25 \mathrm{~km})$ and lower levels $(9-11 \mathrm{~km})$ have high correlation are investigated.

To examine the height of clouds associated with PSCs, the correlation coefficients $(r)$ between the PSC/UC frequencies at two different altitudes are calculated (left panels of Fig. 3). The correlation coefficients greater than about 0.16 are statistically meaningful at a significance level of $99 \%$. The PSC/UC frequencies at $17-23 \mathrm{~km}$ are positively correlated with those at $9-11 \mathrm{~km}$ in June, and those at the respective altitudes in the same altitude range with $r>0.4$. In July, PSC/UC frequencies for 17-25 km are positively correlated with those at other altitudes with the same height range and have $r>0.2$. Correlation coefficients of PSC/UC frequencies at $16-21 \mathrm{~km}$ have a maximum with those around an altitude of $11 \mathrm{~km}$. In August, PSC/UC frequencies at 15$23 \mathrm{~km}$ are not only correlated with those at other altitudes in the same altitude range but also have a stronger correlation $(r>0.3)$ with PSC/UC frequencies at $9-11 \mathrm{~km}$ than those in July. In September, when PSC/UC frequencies are maximized around an altitude of $17 \mathrm{~km}$ (about $10 \%$ ), the correlation coefficients between PSC/UC frequencies around $17 \mathrm{~km}$ with those at $10-11 \mathrm{~km}$ and at $15-19 \mathrm{~km}$ are larger than 0.3 . For June through September, the correlation coefficients of $\mathrm{PSC} / \mathrm{UC}$ frequencies below an altitude of $8 \mathrm{~km}$ and those in the altitude range of $15-25 \mathrm{~km}$ are lower than 0.1 . In summary, PSC/UC frequencies in the altitude range of $15-25 \mathrm{~km}$ are positively correlated with those at $9-11 \mathrm{~km}$ and have a minimum correlation around $14 \mathrm{~km}$ during any month.
On the other hand, the PSC/UC frequencies at 9-11 km are positively correlated with those below $20 \mathrm{~km}$ from July through September. It is important to note that the correlation coefficients of PSC/UC frequencies are even minimized at $13-15 \mathrm{~km}(r<0.2)$ in June, while PSC/UC frequencies at 9$11 \mathrm{~km}$ are significantly correlated with those at $18-21 \mathrm{~km}$ and those at $9-11 \mathrm{~km}$ and have $r>0.4$.

It should be noted that the correlation coefficients between frequencies of $15-25 \mathrm{~km}$ and $9-11 \mathrm{~km}$ are 0.4 at most. Thus, to confirm the relationship between PSCs and UCs, the statistical independency is examined. Figure 4 shows vertical profiles of probability of PSCs with the presence of clouds at 9-11 km (red solid curves) and 5-7 km (red broken curves). Black curves indicate the PSC probability calculated using the entire CALIPSO data set. Cloud occurrence is defined as PSC/UC frequencies greater than $10 \%$. From June through September, the PSC probability with the presence of clouds at $9-11 \mathrm{~km}$ is higher than that calculated using the entire data set. PSC probabilities at $15-25 \mathrm{~km}$ with the presence of clouds at $9-11 \mathrm{~km}$ increment by $19.0,8.6,9.0$ and $7.0 \%$ on average in June, July, August and September, respectively. In September, the increment becomes about half the value of probability of PSC occurrence. These results indicate that PSCs at $15-25 \mathrm{~km}$ are not independent of clouds at 9-11 km. On the other hand, the probability with the presence of clouds at $5-7 \mathrm{~km}$ is nearly equal to that calculated using the entire data set in June through September, which indicates statistical independency between PSCs at $15-25 \mathrm{~km}$ and clouds at $5-7 \mathrm{~km}$. These results from the statistical dependency test confirm the inference that PSCs at $15-25 \mathrm{~km}$ are often observed with clouds at $9-11 \mathrm{~km}$ as obtained from the correlation analyses.

From these features seen in the correlation plots and vertical profiles of cloud occurrence probability, it is inferred that the PSC/UCs at $15-25 \mathrm{~km}$ and those at 9-11 km respectively correspond to simultaneously occurring PSCs and UCs 

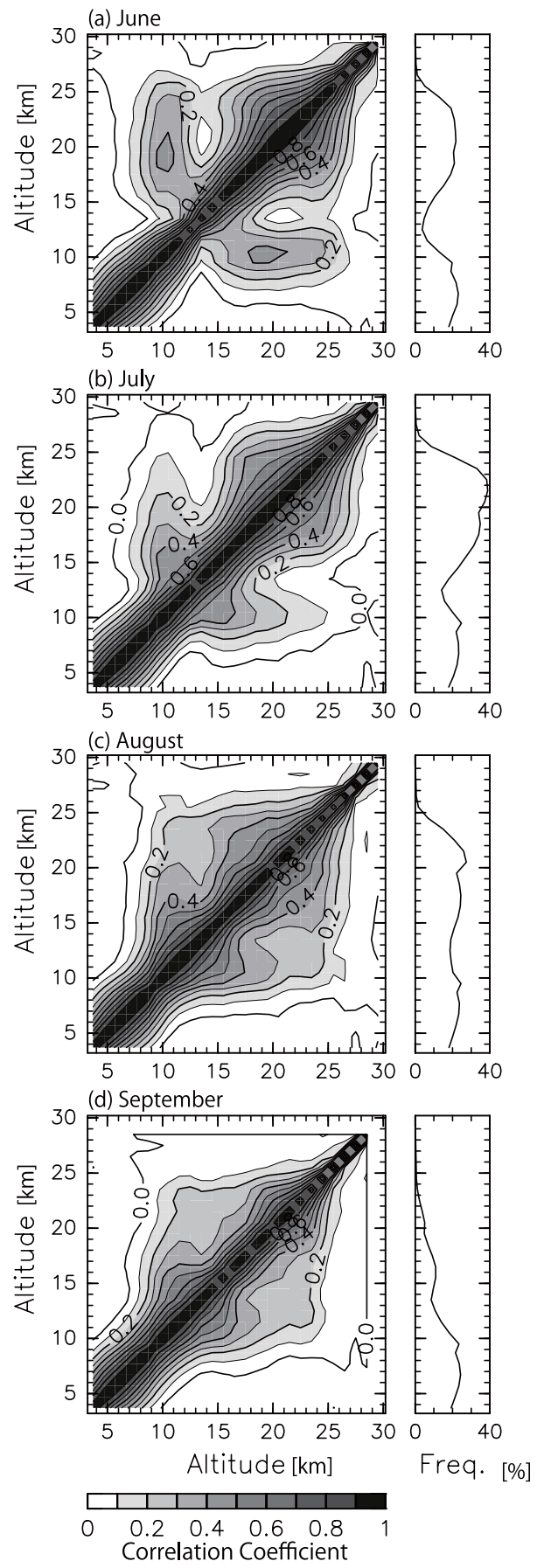

Fig. 3. Correlation coefficients between PSC/UC frequencies at different altitudes for (a) June, (b) July, (c) August, and (d) September from 2007 to 2011 . The correlation coefficients greater than approximately 0.16 are statistically meaningful at a significance level of $99 \%$. The right panel shows PSC/UC frequency as a function of altitude $(\%)$.

as indicated by previous studies (e.g., Wang et al., 2008). However, it should be emphasized here that the tropopause heights can vary in space and time (see Fig. 2) and a typi-
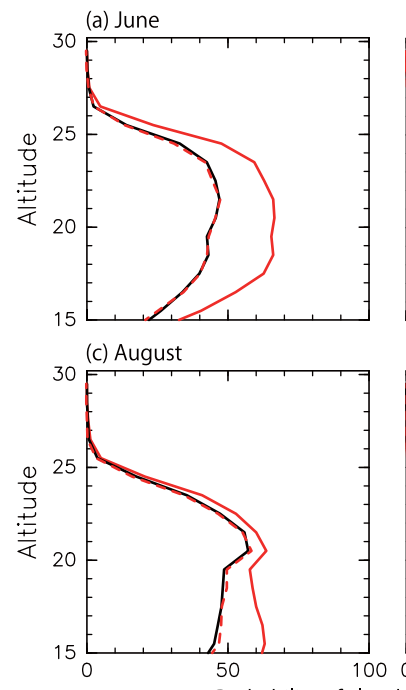

Probability of cloud occurrence [\%]

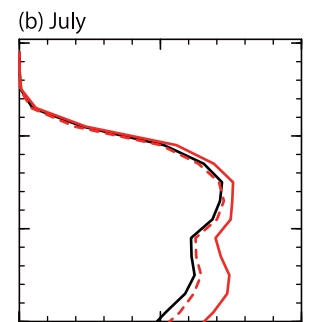

(d) September

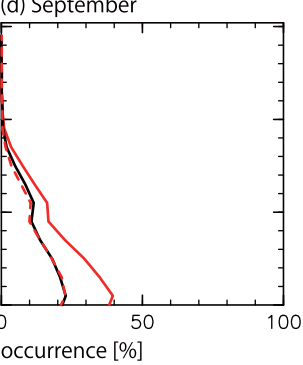

Fig. 4. Vertical profiles of the probability of PSC occurrence for (a) June, (b) July, (c) August, and (d) September from 2007 to 2011. Black solid curves indicate the PSC probability calculated using the entire data set. Red solid (broken) curves indicate the PSC probability in the presence of clouds at 9-11 km (5-7 km).

cal tropopause height in the polar winter is $\sim 8.5 \mathrm{~km}$, which can be lower than the altitudes of UC even when taking into consideration that the tropopause level is modified by atmospheric disturbances. To examine the altitudes of clouds relative to local blended tropopause heights $h_{\mathrm{b}}$, correlation coefficients between PSC/UC frequencies at tropopause-relative altitudes are calculated (Fig. 5). The tropopause-relative altitudes $z_{\mathrm{TP}}$ are defined as $z-h_{\mathrm{b}}$ (i.e., $z \mathrm{TP} \equiv z-h_{\mathrm{b}}$ ), where $z$ is altitude. In this calculation, the correlation coefficients were obtained after linearly interpolating PSC/UC frequency into altitudes relative to $z_{\mathrm{TP}}$. In June, the PSC/UCs at $z_{\mathrm{TP}}=6-$ $13 \mathrm{~km}$, which mostly comprises PSCs, have a positive correlation with those at $z_{\mathrm{TP}}=0-2 \mathrm{~km}(r>0.3)$. The PSC/UC frequencies below the tropopause $(z \mathrm{TP}<0)$ have little correlation with those at $z_{\mathrm{TP}}=6-13 \mathrm{~km}$. Similar characteristics are seen in other months, though they are obscured in September. Because the clouds at $z_{\mathrm{TP}}>6 \mathrm{~km}$ are obviously regarded as PSCs, PSCs are correlated with clouds around or slightly above the tropopause. This fact indicates that it is more appropriate to call these clouds, which occur simultaneously with PSCs, "tropopausal" clouds rather than UCs. Nevertheless, to avoid confusion, in the present study they are referred to as PSCs for PSC/UCs at $15-25 \mathrm{~km}$ and as UCs for those at $9-11 \mathrm{~km}$ in the following analyses.

It is interesting to note that PSC/UC frequencies in the altitude range of $13-15 \mathrm{~km}$, which is where frequency is minimized, have little correlation with those above $18 \mathrm{~km}$ in June and July (Fig. 3a and b). This characteristic is also seen in the analyses based on tropopause-relative altitude: PSC/UC frequencies at $z_{\mathrm{TP}}=3-5 \mathrm{~km}$ do not have significant correlation 

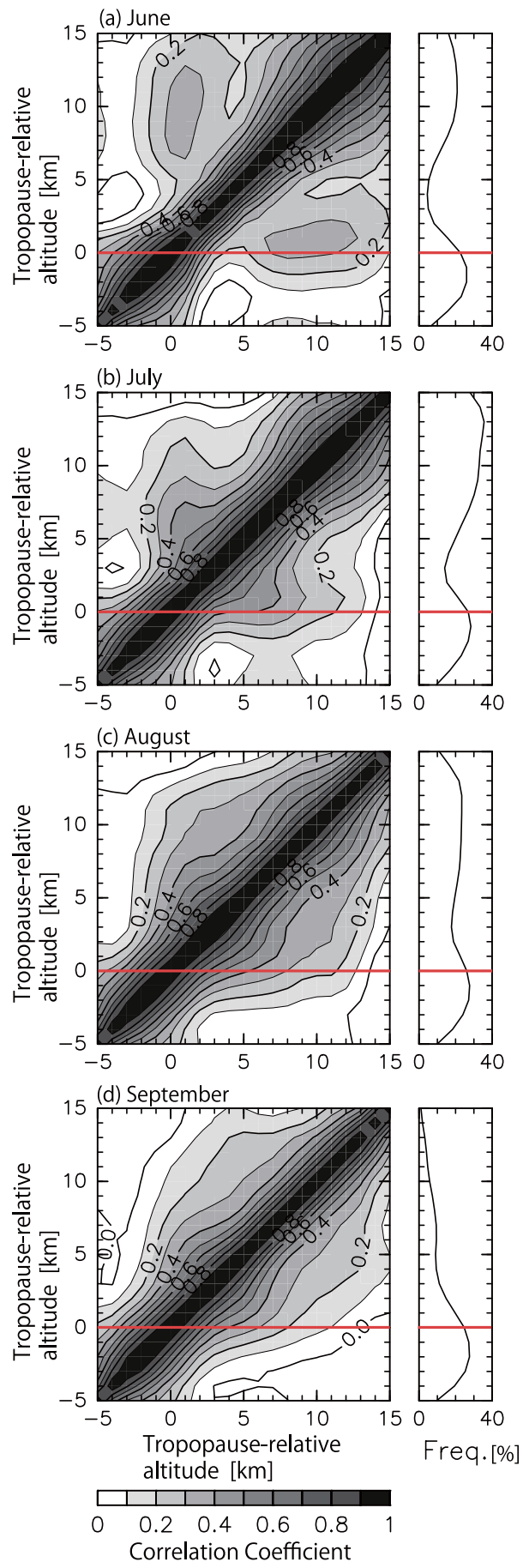

Fig. 5. Correlation coefficients between PSC/UC frequencies as functions of tropopause-relative altitude for June through September from 2007 to 2011 (left). The correlation coefficients greater than approximately 0.16 are statistically meaningful at a significance level of $99 \%$. Red curves indicate $z_{\mathrm{TP}}=0$. The right panel shows PSC/UC frequency as a function of altitude $(\%)$.

with those at $z_{\mathrm{TP}}>8 \mathrm{~km}$ in June and July. This indicates that there is a gap between the UCs and PSCs at approximately $14 \mathrm{~km}$ not only from a view point of the frequency

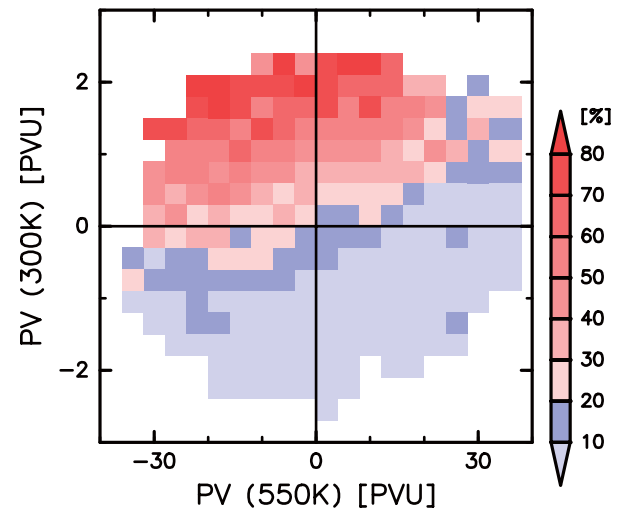

Fig. 6. Probability of the simultaneous occurrence of PSCs at 15$25 \mathrm{~km}$ and UCs at $9-11 \mathrm{~km}$ at $70-75^{\circ} \mathrm{S}$ as a function of PV anomalies from the zonal mean at the $550 \mathrm{~K}$ and $300 \mathrm{~K}$ isentropic surfaces. The size for each bin is $4 \mathrm{PVU} \times 0.3 \mathrm{PVU}$. Time period is June through September from 2007 to 2011.

but also correlation. These results in this subsection suggest that PSCs in the altitude range of $15-25 \mathrm{~km}$ frequently occur simultaneously with UCs at $9-11 \mathrm{~km}$ and not with clouds at approximately $14 \mathrm{~km}$. This cloud gap is particularly clear in June and can be seen in snap shots shown by previous case studies (e.g., Fig. 3 in Wang et al., 2008).

\subsection{Role of tropospheric anticyclones in simultaneous appearance of PSCs and UCs}

Previous studies indicate that PSCs are sometimes observed in association with anticyclonic PV anomalies near the tropopause (Teitelbaum et al., 2001; Kohma and Sato, 2011). Figure 6 shows the probability of the simultaneous occurrence of PSCs and UCs at 70-75 $\mathrm{S}$ in June through September of 2007-2011 as a function of PV anomalies from the zonal mean of $300 \mathrm{~K}(\sim 8 \mathrm{~km})$ and $550 \mathrm{~K}(\sim 23 \mathrm{~km})$ isentropic surfaces. The simultaneous occurrence of PSCs and UCs is defined as when both PSC/UC frequencies at 9-11 km and $15-25 \mathrm{~km}$ are greater than $10 \%$. The PV data are interpolated into the grids of PSC/UC frequency. It is seen that the higher (more anticyclonic) PV anomalies at $300 \mathrm{~K}$ have a higher probability of such a simultaneous occurrence. The averaged probability is higher than $70 \%$ in $300 \mathrm{~K} \mathrm{PV}$ anomalies greater than 1.5 PVU. This result suggests that the anticyclonic PV anomalies near the tropopause $(\sim 300 \mathrm{~K})$ play an important role in the simultaneous occurrence of PSCs and UCs.

Figure 7a shows a composite of the altitude and relativelongitude section of PSC/UC frequencies at $70-75^{\circ} \mathrm{S}$ in June through August of 2007-2011. Here a reference longitude is used at which the PV anomaly from the zonal mean at $72.5^{\circ} \mathrm{S}$ at $300 \mathrm{~K}$ is positively maximized for each day with a value greater than $1.5 \mathrm{PVU}$. The $\mathrm{PSC} / \mathrm{UC}$ frequencies are interpolated linearly by $1.5^{\circ}$ in the longitudinal direction. 
(a)

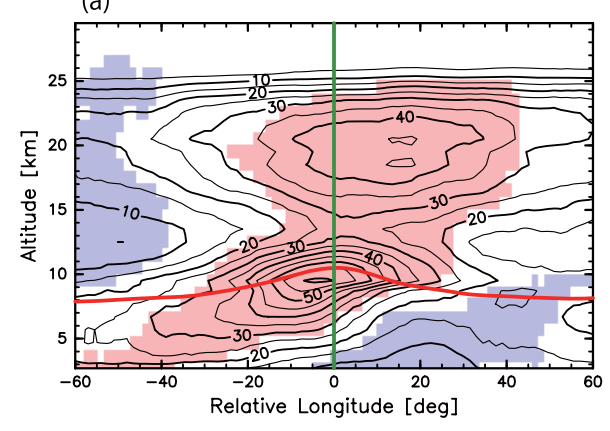

(b)

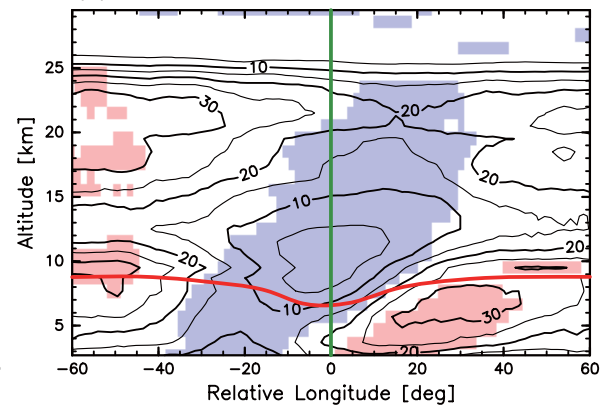

Fig. 7. (a) A composite of relative longitude and altitude of PSC/UC frequency (contours) with a reference longitude (green line) where PV anomalies from the zonal mean are positive and maximized in the zonal direction at $72.5^{\circ} \mathrm{S}$ on a $300 \mathrm{~K}$ isentropic surface for each day, and with values larger than 1.5 PVU. The number of cases used for the composite is 368. Contour intervals are $5 \%$. Red-shaded (blue-shaded) areas are the regions with PSC/UC frequencies higher (lower) than their averaged values and with significance levels greater than $95 \%$. The red thick curve indicates the composite of the blended tropopause. (b) The same as (a) but when using a reference longitude where PV anomalies from the zonal mean are negative and minimized in the zonal direction at $72.5^{\circ} \mathrm{S}$ on a $300 \mathrm{~K}$ isentropic surface for each day, and with values smaller than -1.5 PVU. The number of cases used for the composite is 374 . Time period for both plots is June through August from 2007 to 2011 .

The observations in September are excluded from the analyses as the averaged PSC frequencies at $15-25 \mathrm{~km}$ are significantly lower in September than those in the other months (see the right panels of Fig. 3). Significantly high frequencies are seen around the dynamical tropopause denoted by a red curve $(\sim 9-10 \mathrm{~km})$ near the reference longitude. High frequencies are also observed at $15-25 \mathrm{~km}$ in a similar longitude range. These features suggest that the simultaneous occurrence of PSCs and UCs is associated with anticyclones near the tropopause. It should be particularly noted that the frequencies at $5-12 \mathrm{~km}$ are also significantly high in the westward regions of anticyclones. Previous studies (Wang et al., 2008; Adhikari et al., 2010; Achtert et al., 2012) found a significant connection between PSCs and tropospheric "deep and high" clouds. The high frequencies westward of an anticyclone may correspond to these "deep and high" clouds shown in the previous studies. This point is discussed in detail in Sect. 4.

It should be noted that the dynamical tropopause is slightly elevated around the reference longitude of the anticyclonic PV anomaly, which is consistent with a theoretical study by Wirth (2000). UCs are observed around and even slightly above the elevated tropopause (i.e., in the stratosphere). This fact again indicates that it is more appropriate to call them "tropopausal" clouds rather than UCs.

To clearly show that tropospheric anticyclones are important for the simultaneous occurrence of PSCs and UCs, an analysis similar to Fig. 7a, but for cyclonic PV anomalies, was performed and is shown in Fig. 7b. The reference longitude is determined by a PV anomaly from the zonal mean at $72.5^{\circ} \mathrm{S}$ at $300 \mathrm{~K}$ that is minimized for each day with a value smaller than -1.5 PVU. PSC and UC frequencies in the altitude range of $8-24 \mathrm{~km}$ are significantly low near the cyclone. This is likely due to the fact that cyclonic PV anomalies are associated with positive temperature anomalies above this range (e.g., Kohma and Sato, 2011). These results suggest that the simultaneous occurrence of PSCs and UCs is closely related to anticyclones, rather than cyclones. It is also noteworthy that the tropopause is lowered near the cyclone (i.e., relative longitude $\sim 0$ ), which is consistent with the study by Wirth (2000).

Figure 8a shows a typical example of a concurrence of PSCs and UCs over the Antarctic Peninsula along the CALIPSO orbit on 2 August 2008. PSCs and UCs are observed along the orbit from $\left(62^{\circ} \mathrm{S}, 67^{\circ} \mathrm{W}\right)$ to $\left(78^{\circ} \mathrm{S}, 93^{\circ} \mathrm{W}\right)$ in the altitude range of $15-25 \mathrm{~km}$ and below $12 \mathrm{~km}$. Few clouds are observed around $14 \mathrm{~km}$, which corresponds to the cloud gap between the PSC and UC regions as statistically seen in Fig. 3a. Figure 8b is a PV map at $300 \mathrm{~K}$ isentropic surface indicated by red contours, geopotential heights at $300 \mathrm{hPa}(\sim 8 \mathrm{~km})$ indicated by colored shading, and the satellite orbit which is indicated by a thick broken curve. An anticyclone with a horizontal scale larger than $3000 \mathrm{~km}$ is located over the Antarctic Peninsula. The longitude-pressure section of the geopotential height anomalies from the zonal mean at $65^{\circ} \mathrm{S}$ is shown in Fig. $8 \mathrm{c}$. It is clear that an anomaly maximum corresponding to the anticyclone at $65^{\circ} \mathrm{W}$ extends vertically to $30 \mathrm{hPa}(\sim 22 \mathrm{~km})$. Temperatures are minimized near the maximum geopotential height and lower than $185 \mathrm{~K}$ at $20-60 \mathrm{hPa}$.

It is examined whether the anticyclonic PV anomalies near the tropopause can cause low temperatures in the altitude range of $15-25 \mathrm{~km}$. First, we considered an ideal situation in which a delta function-like anticyclonic PV anomaly is present at the tropopause level in the vertical direction. This situation is similar to an anticyclonic PV anomaly at the upper boundary in Eady's baroclinic instability theory (e.g., Vallis, 2006). The vertical penetration of a temperature 


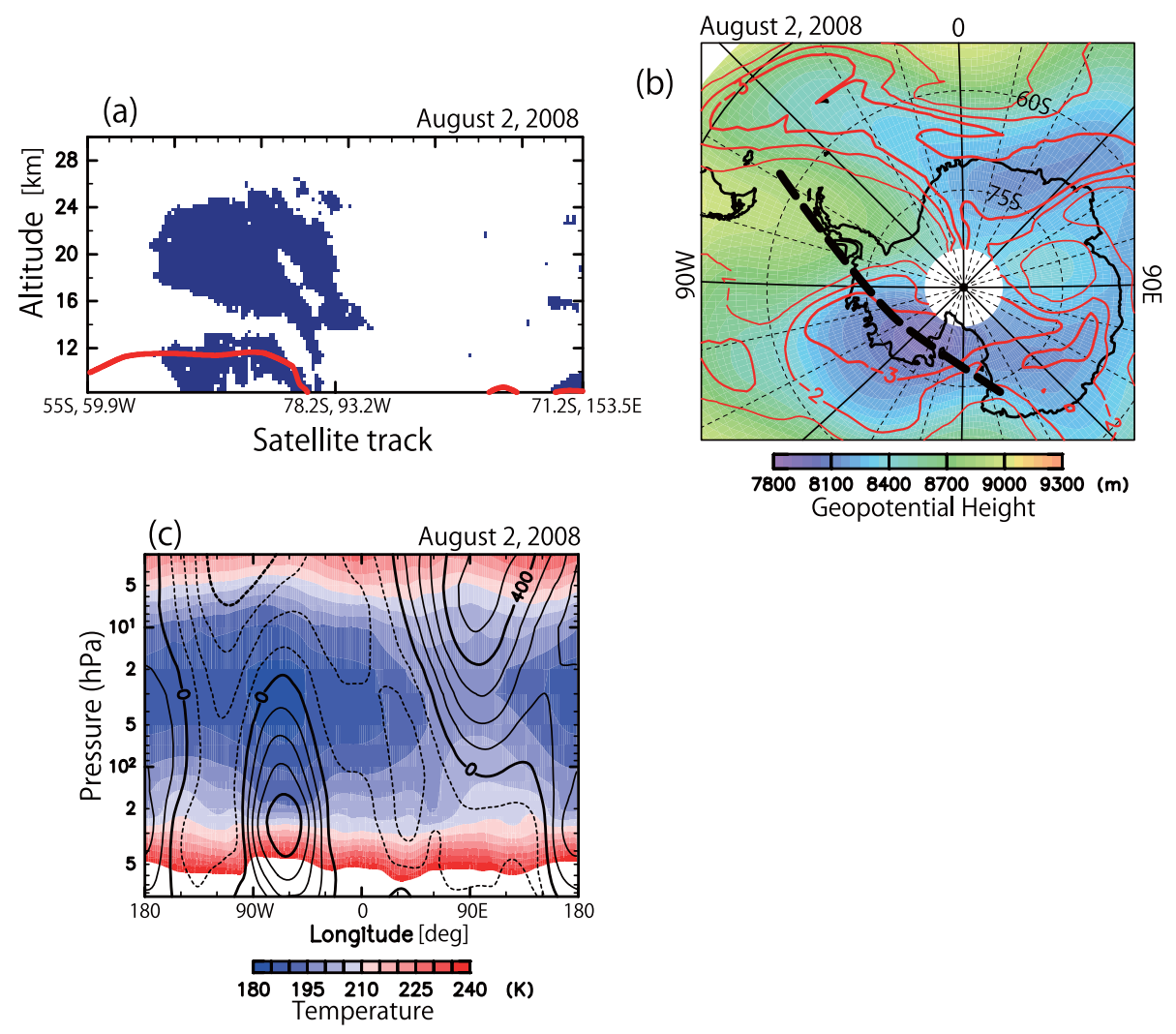

Fig. 8. (a) A horizontal and height section of the location of PSC/UC flags (blue color) along the CALIPSO orbit on 2 August 2008. The red curve indicates the tropopause heights. (b) A polar stereo projection map of geopotential heights (in color; unit is $\mathrm{m}$ ) at $300 \mathrm{hPa}$ and PV (red contour; unit is PVU) on a $300 \mathrm{~K}$ isentropic surface. Contour interval is 1 PVU. The broken thick curve indicates the satellite track in (a). (c) A longitude and pressure section of geopotential height anomalies from the zonal mean (contours; unit is m) and temperatures (in color; unit is $\mathrm{K}$ ) at $65^{\circ} \mathrm{S}$. Contour interval is $100 \mathrm{~m}$. Broken contours indicate negative values.

anomaly above the location of the PV anomaly is obtained at the Rossby height (Hoskins et al., 1985). The Rossby height $H_{\mathrm{R}}$ is defined as $f L / 2 \pi N$, where $f$ is the Coriolis parameter, $N$ is static stability, and $L$ is horizontal wavelength $(=2 \pi / k$, $k$ is horizontal wavenumber). In winter high latitudes, a typical $H_{\mathrm{R}}$ value is estimated at about $5 \mathrm{~km}$ in the lowermost stratosphere, and $N=0.02 \mathrm{~s}^{-1}$ and $L=5000 \mathrm{~km}$ are used. As the tropopause is climatologically located around a height of $8.5 \mathrm{~km}$, temperature anomalies should only have significant amplitudes up to $14 \mathrm{~km}$. Even if we consider the elevation of the tropopause by about $2 \mathrm{~km}$ associated with the anticyclone as seen in Fig. 7, the vertical extent cannot reach a height of $16 \mathrm{~km}$. Thus, the existence of PSCs in the altitude range of $16-25 \mathrm{~km}$ is not very attributable to anticyclonic PV anomalies confined to the tropopause.

An anticyclonic PV anomaly with a finite depth was taken into consideration. An analysis was conducted on modified PV distributions (Lait, 1994) using ERA-Interim data on 2 August 2008. The definition of modified PV, $\Pi$, is as follows:

$\Pi=P\left(\theta / \theta_{0}\right)^{-9 / 2}$, where $P$ and $\theta$ are Ertel's PV and potential temperature, respectively. Here, $\theta_{0}$ is $500 \mathrm{~K}$. Multiplying $\theta^{-9 / 2}$ compensates an exponential increase in PV with altitude, and makes it easy to examine the vertical structures of the polar vortex and atmospheric disturbances. Figure 9a shows a geographical latitude-pressure section of the modified $\mathrm{PV}$ at $60^{\circ} \mathrm{W}$. Low modified PV is seen in higher latitudes in the stratosphere while modified PV in lower latitudes is high. However it is difficult to assess the depth of the PV anomaly near the tropopause due to the change of static stability, which is included in the PV formula across the tropopause.

Another convenient approach to the assessment of the PV anomaly's depth is to make use of the equivalent latitudes $y_{\mathrm{e}}$ (that is, dynamical latitudes defined based on PV) (Butchart and Remsberg, 1985). It should be noted that the range of equivalent latitudes at each isentropic surface is $90^{\circ} \mathrm{N}-90^{\circ} \mathrm{S}$ by definition. The relationship between modified PV and equivalent latitudes on several isentropic planes is shown in Fig. 9b. One-to-one correspondence between the modified $\mathrm{PV}$ and equivalent latitudes is clearly shown for each isentropic surface. Thus, the equivalent latitudes are regarded as normalized PVs in isentropic surfaces. The unperturbed PV 

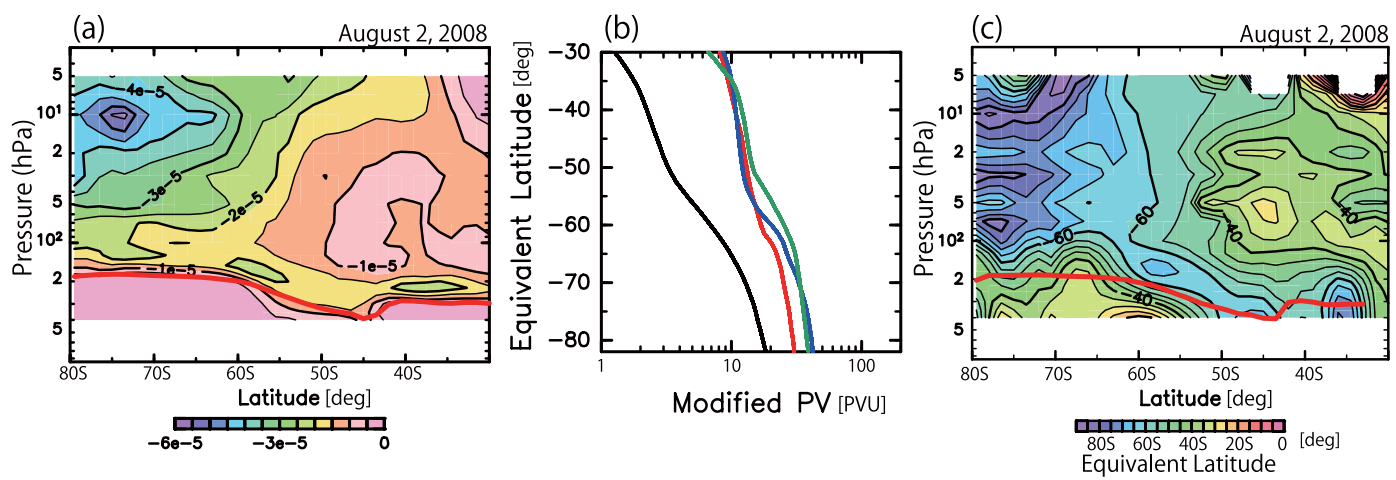

Fig. 9. (a) A geographical latitude and pressure section of modified PV at $60^{\circ} \mathrm{W}$. The red curve shows the tropopause on 2 August 2008 . (b) Equivalent latitudes as functions of absolute value of modified PV on 300 (black), 400 (red), 500 (blue), and $600 \mathrm{~K}$ (green) isentropic surfaces. (c) The same as (a) but for equivalent latitudes.

isolines are usually oriented zonally near the polar vortex, which is primarily due to planetary vorticity. Because Ertel's $\mathrm{PV}$ and a modified PV are materially conserved in adiabatic and inviscid flow, the equivalent latitude indicates which latitude the air parcel originally comes from. Thus, small $y_{\mathrm{e}}$ values indicate the intrusion of air from equatorward regions, i.e., anticyclonic PV intrusion.

Figure $9 \mathrm{c}$ shows a geographical latitude-pressure section of the equivalent latitudes at $60^{\circ} \mathrm{W}$. An important feature is that the air at equivalent latitudes lower than $60^{\circ} \mathrm{S}$ intrudes into a geographical latitude of $67^{\circ} \mathrm{S}$ in the troposphere with a vertical extent of up to $100 \mathrm{hPa}$ (about $15 \mathrm{~km}$ ) (see contour lines of $y_{\mathrm{e}}=60^{\circ} \mathrm{S}$ ). As mentioned above, equivalent latitudes that are lower than geographical latitudes indicate anticyclonic PV intrusion. When considering a height of $15 \mathrm{~km}$ as the upper limit of tropospheric disturbances, the vertical penetration is estimated at $20 \mathrm{~km}$ using the Rossby height $(\sim 5 \mathrm{~km})$ for values of $N=0.02 \mathrm{~s}^{-1}$ and $L=5000 \mathrm{~km}$. Thus, an anticyclonic disturbance with a large horizontal scale and tall structure, such as those observed in Figs. 7, 8b, and 8c, can cause low temperatures in the entire altitude range where PSCs and UCs are observed. These characteristics of large horizontal and vertical spatial structures suggest that the anticyclonic disturbances are likely due to blocking highs in the troposphere (e.g., Schwierz et al., 2004).

It should be noted that the cloud gap near $14 \mathrm{~km}$, which can be seen in Fig. 8a, cannot be explained by this mechanism alone. An additional factor must be considered. Mixing processes are a plausible cause for the supply of $\mathrm{H}_{2} \mathrm{O}$-rich air from the troposphere to the stratosphere. Based on trajectory analyses, Pfister et al. (2002) showed that this tropospherestratosphere exchange extends to about $13 \mathrm{~km}$ in the Arctic winter and that mixing ratios of $\mathrm{H}_{2} \mathrm{O}$ can be significantly higher than a typical value of $5 \mathrm{ppmv}$ in the stratosphere. Because such a humid air supply from the troposphere reaches up to a maximum altitude of $13 \mathrm{~km}$, the level of minimum water vapor (hygropause) is located around $14 \mathrm{~km}$ (Chiou et al., 1997). This is likely one reason why only a few clouds are formed around $14 \mathrm{~km}$.

\subsection{Longitudinal dependencies of blocking highs and simultaneous occurrence of PSCs and UCs}

In this section, the occurrence frequency of blocking highs is estimated using daily mean ERA-interim data based on a method similar to the criteria suggested by Schwierz et al. (2004). First, the analyzed area is limited to a latitude range of $55-85^{\circ} \mathrm{S}$, where PSCs are mainly observed. Second, equivalent latitudes that are vertically averaged for isentropic levels of 300-320 K are obtained for each day, since blocking highs have a tall structure (Schwierz et al., 2004). Third, the regions surrounded by equivalent latitude contours of $55^{\circ} \mathrm{S}$ and/or a geographic latitude circle of $55^{\circ} \mathrm{S}$ are obtained as candidates for blocking highs. Last, the blocking highs are selected from these candidates by imposing the following two criteria: the horizontal area is greater than $3 \times 10^{6} \mathrm{~km}^{2}$, and the southernmost geographical latitude of the candidate blocking high is higher than $65^{\circ} \mathrm{S}$. The threshold of the former criterion was chosen to examine the spatial scales corresponding to $H_{\mathrm{R}}$ of approximately $4 \mathrm{~km}$ at $65^{\circ} \mathrm{S}$. The latter criterion ensures that the areal extent of a blocking high covers high latitudes where the PSCs are frequently observed.

Schwierz et al. (2004) originally used four types of thresholds for amplitude, spatial scale, overlap, and duration in order to track blocking highs, while the present study uses only two thresholds for amplitude and spatial scale. The "overlap" parameter corresponds to the spatial overlap between blocking highs of one time grid and the next time grid. Using additional thresholds of 0.4 for "overlap" and 2 days for "duration" for blocking detection makes the total number of blocking highs decrease by about $20 \%$ in the present analysis. However, the spatial and temporal distribution of blocking highs defined using these two additional parameter thresholds was quite similar to that when they were not used (not shown). Thus, in order to provide a sufficient number of 
Table 2. (a) Percentage of simultaneously occurring PSCs at $15-20 \mathrm{~km}$ and UCs at $9-11 \mathrm{~km}$ that are in the presence of blocking highs. (b) The same as (a) but for PSCs at 20-25 km and UCs at 9-11 km.

\begin{tabular}{llllll}
\hline & 2007 & 2008 & 2009 & 2010 & 2011 \\
\hline (a) PSC $(z=15-20 \mathrm{~km}) \&$ UC $(z=9-11 \mathrm{~km})$ & $47 \%$ & $45 \%$ & $41 \%$ & $39 \%$ & $48 \%$ \\
(b) PSC $(z=20-25 \mathrm{~km}) \&$ UC $(z=9-11 \mathrm{~km})$ & $50 \%$ & $43 \%$ & $43 \%$ & $37 \%$ & $39 \%$ \\
\hline
\end{tabular}

cases of blocking highs for the statistical analyses, the "overlap" and "duration" parameters were not used in the present analyses.

The obtained blocking high covers about $15 \%$ of the latitudinal area of $55-82^{\circ} \mathrm{S}$ in June through September on average. This value should be similar to the percentage of simultaneous occurrence of PSCs and UCs in the presence of blocking highs if the simultaneous occurrence were independent of blocking highs. In fact, the concurrence of PSCs and UCs is related to the presence of blocking highs, as discussed in the following.

The altitude region of $15-25 \mathrm{~km}$ is separated into two layers, since the spatial resolutions of the CALIPSO Level 1B data above and below $20 \mathrm{~km}$ are different. Table $2 \mathrm{a}$ shows the percentage of simultaneously occurring PSCs and UCs that accompany blocking highs in the five austral winters in a region only inside the polar vortex on a $500 \mathrm{~K}$ isentropic surface. Here, the edge of the polar vortex is determined based on PV (Nash et al., 1996). Observations in the latitude range of $75-82^{\circ} \mathrm{S}$ are not used in the following analyses, since PSCs are almost always observed from July to August due to the presence of sufficiently low temperatures regardless of presence/non-presence of a blocking high. The simultaneous occurrence of PSCs and UCs is defined as when both PSC/UC frequencies at 9-11 km and 15$20 \mathrm{~km}$ are greater than $10 \%$. As shown in Table $2 \mathrm{a}$, it was found that $44 \%$ of such concurrent occurrences are associated with blocking highs. This value is slightly changed by the thresholds used in the blocking high identification process, and is about three times as large as the coverage of the blocking highs $(15 \%)$, which indicates that the concurrence of PSCs and UCs is likely to be accompanied by blocking highs. Table 3 a shows the probability of the simultaneous occurrence of PSCs and UCs when the blocking high is present and not present. The probability of such an occurrence with the presence of a blocking high is three times larger than that with the absence of a blocking high. These facts suggest that blocking highs strongly affect the simultaneous occurrence of PSCs and UCs.

The same analyses for PSCs at 20-25 km and UCs at 9$11 \mathrm{~km}$ were performed (Table $2 \mathrm{~b}$ and Table $3 \mathrm{~b}$ ). The results of each case are quite similar to those in Table $2 \mathrm{a}$ although the values in Table $2 b$ are slightly smaller than those in Table $2 \mathrm{a}$.

The frequency of the simultaneous occurrence of PSCs at 15-25 km and UCs at 9-11 km (black solid curves) and that
Table 3. (a) Probabilities of the simultaneous occurrence of PSCs at $15-20 \mathrm{~km}$ and UCs at $9-11 \mathrm{~km}$ in the presence and absence of blocking highs. (b) The same as (a) but for PSCs at 20-25 km and UCs at $9-11 \mathrm{~km}$.

\begin{tabular}{lrrrrr}
\hline (a) & 2007 & 2008 & 2009 & 2010 & 2011 \\
\hline Blocking high & $39 \%$ & $41 \%$ & $39 \%$ & $33 \%$ & $37 \%$ \\
No blocking high & $10 \%$ & $10 \%$ & $12 \%$ & $9 \%$ & $10 \%$ \\
\hline (b) & 2007 & 2008 & 2009 & 2010 & 2011 \\
\hline Blocking high & $36 \%$ & $37 \%$ & $38 \%$ & $21 \%$ & $32 \%$ \\
No blocking high & $8 \%$ & $10 \%$ & $10 \%$ & $6 \%$ & $12 \%$ \\
\hline
\end{tabular}

of blocking highs (red solid curves) is shown as a function of longitude for the five austral winters in Fig. 10. The frequency of blocking highs is calculated for the region only inside the polar vortex on a $500 \mathrm{~K}$ isentropic surface in the middle stratosphere. The longitudinal variations of frequency of blocking highs resemble those of the simultaneous occurrence of PSCs and UCs. The correlation coefficients increase to $0.77,0.72,0.35,0.81$ and 0.25 in $2007,2008,2009,2010$ and 2011, respectively. These results indicate that the tropospheric blocking highs provide preferable conditions for the simultaneous occurrence of PSCs and UCs inside the stratospheric polar vortex.

Previous studies (e.g., Kohma and Sato, 2011) showed that PSCs are more frequently observed over the Antarctic Peninsula than in the other longitudes of $55-82^{\circ} \mathrm{S}$. Several studies have indicated that a cold phase of temperature fluctuations associated with mountain waves originating from the Antarctic Peninsula increases the formation of ice particles, on which the heterogeneous nucleation of NAT particles can occur (Carslaw et al., 1998; Höpfner et al., 2006; Eckermann et al., 2009). Because NAT particles have a higher equilibrium temperature than the ice frost point, the occurrence frequency of PSCs over the Antarctic Peninsula is able to rise. An important and interesting feature is shown in Fig. 10. The frequency of PSC occurrence is large in longitudinal regions $\left(80-120^{\circ} \mathrm{W}\right)$ and even windward of the Antarctic Peninsula $\left(60-70^{\circ} \mathrm{W}\right)$. It is not likely that orographic gravity waves cause low temperatures in such a region. The PSC frequency at $15-25 \mathrm{~km}$ that is associated with blocking highs in the latitude range of $70-75^{\circ} \mathrm{S}$ is approximately $32 \%$, which is significantly higher than the mean PSC frequency $(17 \%)$. Therefore, blocking highs are a strong candidate to cause 

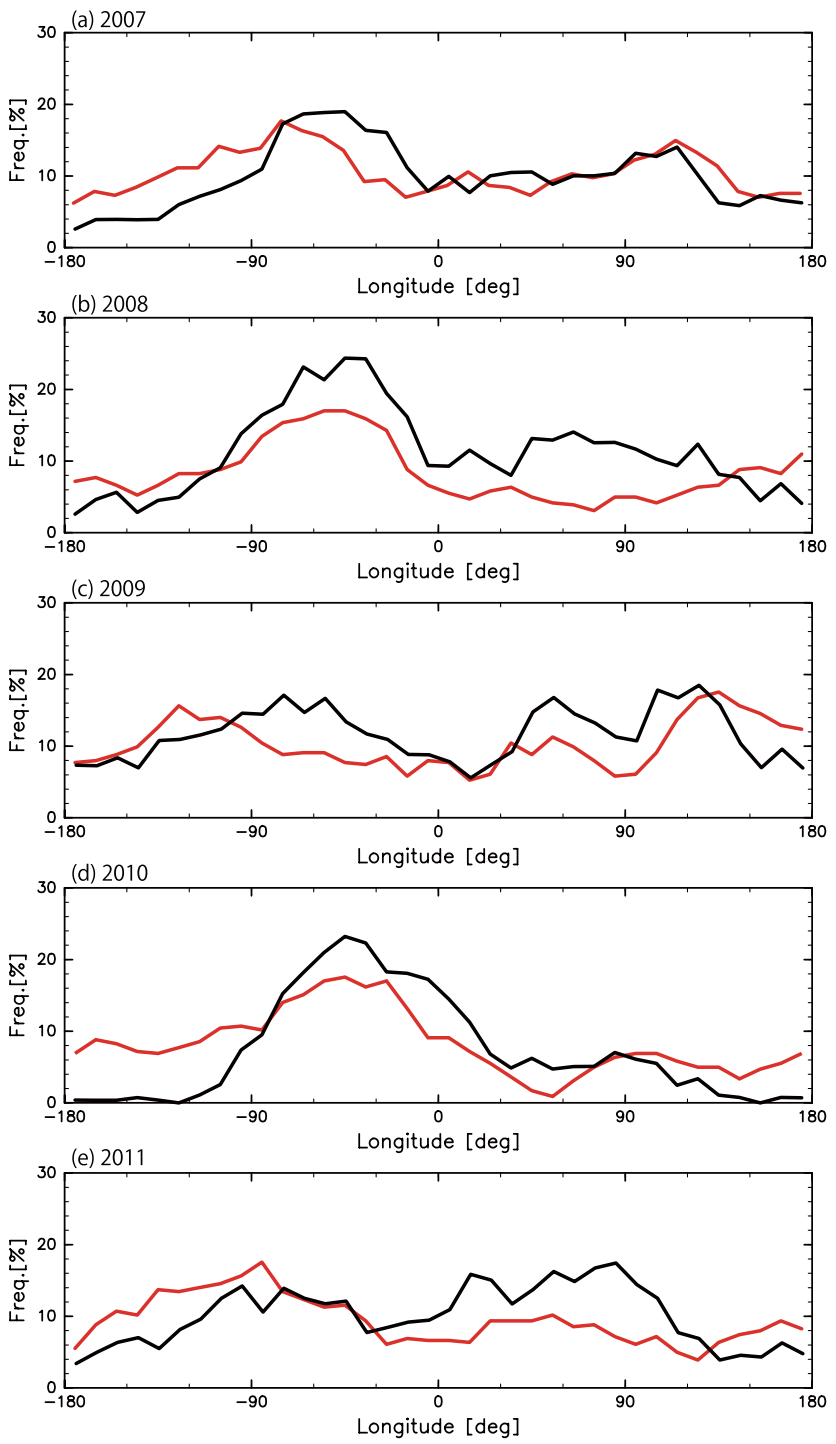

Fig. 10. Black curves show frequencies of simultaneous observations of PSCs in the altitude range of $15-25 \mathrm{~km}$ and UCs at 9-11 km as functions of longitude from June through September (a) 2007, (b) 2008, (c) 2009, (d) 2010 and (e) 2011. Red solid curves show frequencies of blocking highs calculated for the region only inside the polar vortex on a $500 \mathrm{~K}$ isentropic surface.

high PSC frequencies in the windward regions of the Antarctic Peninsula (Fig. 10).

\subsection{Effects of blocking highs on PSC composition}

Adhikari et al. (2009) showed that ice PSCs are frequently observed in association with tropospheric cloud systems, especially in the late PSC season. Figure 11a shows PSC frequencies as functions of altitude and relative longitude to anticyclones at $70-75^{\circ} \mathrm{S}$ in August for the five austral winters. Significant results were not obtained in September and October due to the insufficient number of cases of PSCs above an altitude of $15 \mathrm{~km}$. Figure 11b-e show longitude-altitude sections of the fraction (\%) of PSCs categorized into PSC composition (i.e., STS, Mix 1, Mix2 and Ice). It is clear that Mix2 clouds are the most dominantly occurring clouds, which is consistent with the climatological PSC composition in August shown by Pitts et al. (2009).

The areas of interest are the relative longitudes where the fraction of each PSC composition is largest. It should be noted that the dominant mean flows are eastward in the polar stratospheric winter. The proportions of Mix 1 and Mix2 PSCs are higher to the east of the reference longitude, namely, to the leeward side of anticyclonic PV. The proportion of Ice PSCs is maximized around the reference longitude (i.e., zero relative longitude). The proportion of STS PSCs is higher to the windward regions of anticyclonic PV than that of the leeward side.

The dependence of PSC composition on the relative location between the anticyclone center and PSCs as observed in Fig. 11 may be understood in the same manner of the ice-seeding process by gravity waves (Carslaw et al., 1998; Höpfner et al., 2006; Eckermann et al., 2009) but is alternatively understood by anticyclones in the present case. Note that this inference is consistent with the study by Larsen et al. (2004), which showed that solid particles are strongly affected by synoptic-scale temperature histories rather than local temperatures in Arctic early winter.

\section{Discussion}

Wang et al. (2008) suggested that tropospheric cyclones possibly cause the concurrence of PSCs and UCs. They speculated that the vertical material transport associated with tropospheric cyclones is responsible for the occurrence of PSCs and that the intense cyclones are typically accompanied by high and deep clouds above $7 \mathrm{~km}$. However, because climatological convective available potential energy (CAPE) in the polar region is much less than that in the tropics (RiemanCampe et al., 2009), it is rare that convection in the polar region is sufficiently strong and deep to overshoot the tropopause and transport water-rich air from the troposphere into the stratosphere. Moreover, Pan and Munchak (2012) showed that cloud tops that are above the tropopause related to deep convection are located at $2.5 \mathrm{~km}$ above the tropopause at most, even in the tropics. This fact also suggests that it is quite difficult for the tropospheric deep convection in the polar region to cause direct material transport from the tropopause level $(\sim 8.5 \mathrm{~km})$ to altitudes where PSCs are often observed $(\sim 20 \mathrm{~km})$.

We examined the meteorological conditions for the two cases of PSCs and UCs shown by Wang et al. (2008) for 25 July 2006 and by Adhikari et al. (2010) for 25 June 2006. Figure 12 shows the distribution of PSC/UC flags of the horizontal and height sections from the CALIPSO Level 1B data and polar stereo projection maps of geopotential height 

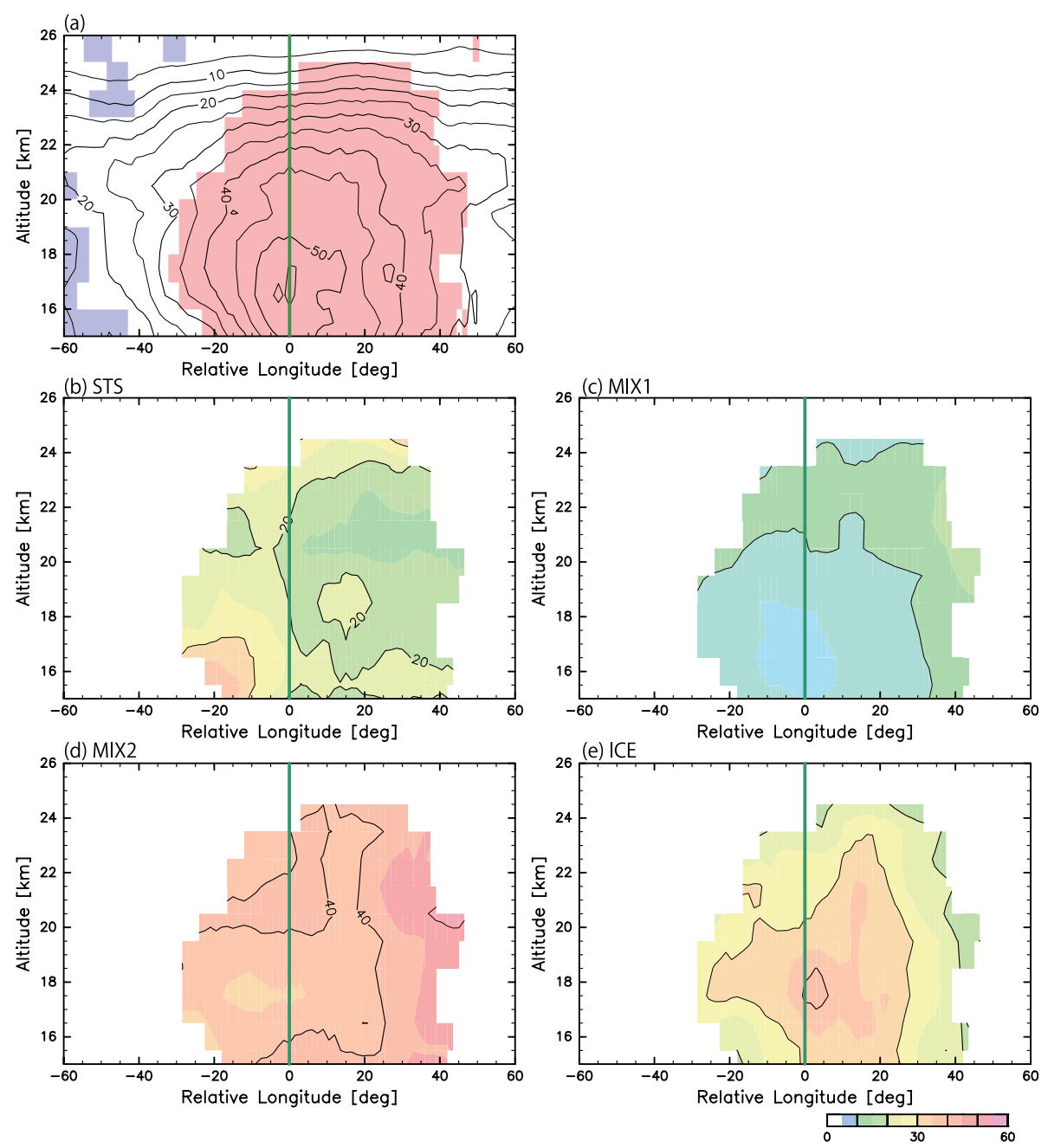

Fig. 11. (a) The same as Fig. 7 but for August of 2007-2011. The number of cases used for the composite is 107. (b-d) The same as (a) but for the proportion for each PSC composition: (b) STS, (c) Mix1, (d) Mix2 and (e) Ice. Coloring corresponds to the significantly high $\mathrm{PSC} / \mathrm{UC}$ frequency regions in (a). Contour interval is $20 \%$.

at $300 \mathrm{hPa}$ for the two cases. On 25 June, two clusters of PSCs are observed above the UCs in the altitude range of $15-24 \mathrm{~km}$ along the orbit paths of $\left(70^{\circ} \mathrm{S}, 75^{\circ} \mathrm{W}\right)$ to $\left(80^{\circ} \mathrm{S}\right.$, $\left.30^{\circ} \mathrm{W}\right)$ and $\left(68^{\circ} \mathrm{S}, 55^{\circ} \mathrm{E}\right)$ to $\left(81^{\circ} \mathrm{S}, 0^{\circ}\right)$. The cloud tops of UCs are slightly above the tropopause, which is consistent with Fig. 12. The two UCs are accompanied by uppertropospheric ridges, while the regions with no UCs between the ridges correspond to the tropospheric trough. On 25 July, PSCs and UCs are simultaneously observed over the Weddell Sea. Additionally, there is a tropospheric anticyclone over the Weddell Sea, which corresponds well with Fig. 8b. Therefore, both cases of simultaneous occurrences of PSCs and UCs shown in the previous studies are associated with tropospheric anticyclones, not with cyclones. It is also noticeable that elevated tropopause heights are observed below the PSCs in both cases, which is consistent with the study of Fromm et al. (2003).

\section{Summary and concluding remarks}

In this study, by using satellite lidar data and reanalysis data from the five austral winters of 2007-2011, the simultaneous occurrence of PSCs and UCs in the Southern Hemisphere has been examined. In particular, the role of blocking highs was thoroughly investigated.

A correlation analysis has shown that PSCs in an altitude range of $15-25 \mathrm{~km}$ are positively correlated with UCs in an altitude range of $9-11 \mathrm{~km}$. The cloud frequency below $8 \mathrm{~km}$ was slightly correlated with those of PSCs. These results were confirmed by a statistical independence test. From the analyses using altitude relative to the local tropopause height, it was also shown that the UCs concurrently appearing with PSCs are located around and slightly above the tropopause.

The simultaneous occurrence frequencies of the PSCs and UCs were significantly high when blocking highs were present in the troposphere. The blocking high had a deep 
(a) June 25, 2006

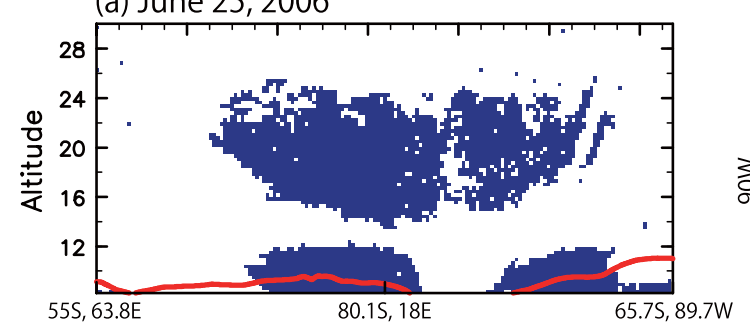

(b) June 25, $2006 \quad 0$

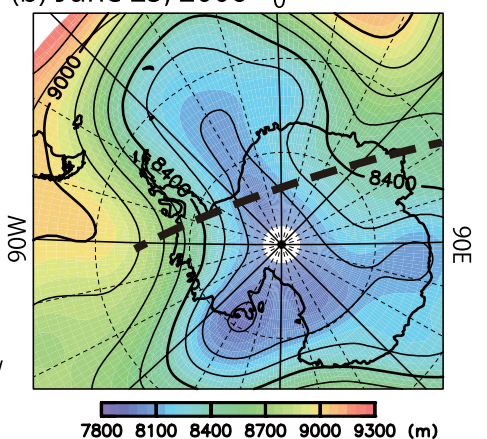

(d) July 25, 2006
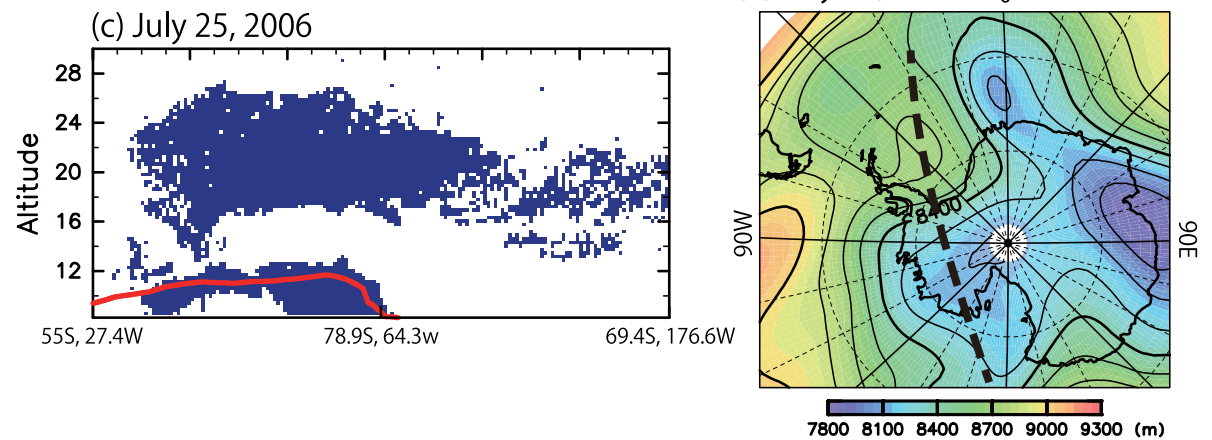

Fig. 12. (a) The distribution of PSC/UC flags (blue color) in the horizontal and height sections along the CALIPSO on 25 June 2006 . A red curve indicates the blended tropopause height. (b) A polar stereo projection plot of geopotential heights (in color; unit is $\mathrm{m}$ ) at $300 \mathrm{hPa}$. The broken thick curve indicates the satellite track in (a). (c) and (d) the same as (a) and (b), respectively, but for 25 July 2006.

PV anomaly structure that caused the negative temperature anomalies to vertically extend up to $20 \mathrm{~km}$, which is where PSCs are commonly observed. In the region inside the polar vortex on the $500 \mathrm{~K}$ isentropic surface, the frequencies of blocking highs and of the simultaneous occurrence of PSCs and UCs exhibit similar longitudinal variations. Furthermore, the higher occurrence frequency of PSCs to the windward region of the Antarctic Peninsula than any other regions can be explained by the distribution of blocking frequency.

It has also been shown that dominant PSC composition depends on the longitude relative to the center of blocking highs. Ice PSCs are relatively rich above the blocking highs, while the proportions of NAT-rich and STS-rich PSCs increase to leeward and windward regions of blocking highs, respectively. Höpfner et al. (2006) explained the ice-seeding process by discussing the extremely low temperature fluctuations associated with gravity waves. The present result suggests that blocking highs also lead to a similar ice-seeding effect.

A cloud gap was found around $14 \mathrm{~km}$, which may also be related to the enhancement of $\mathrm{H}_{2} \mathrm{O}$ mixing ratios in the lowermost stratosphere due to cross-tropopause mixing. This issue should be examined in future studies.
Acknowledgements. The CALIPSO data product was provided by the NASA Langley Atmospheric Science Data Center. ERA-Interim data were provided by the European Center for Medium-Range Weather Forecast (ECMWF). The figures were produced by the software tools developed under the GFD Dennou Club Davis project. We thank Y. Tomikawa and S. Alexander for their help to the treatment of the data set. This study was supported by Grantin-Aid Scientific Research (B) 22340134 and by Grant-in-Aid for Research Fellow (23-9377) of the JSPS. This manuscript was proofread by a proofreading/editing assistant of the Global COE program From the Earth to "Earths".

Edited by: D. Knopf

\section{References}

Achtert, P., Karlsson Andersson, M., Khosrawi, F., and Gumbel, J.: On the linkage between tropospheric and Polar Stratospheric clouds in the Arctic as observed by space-borne lidar, Atmos. Chem. Phys., 12, 3791-3798, doi:10.5194/acp-12-3791-2012, 2012.

Adhikari, L., Wang, Z., and Liu, D.: Microphysical properties of Antarctic polar stratospheric clouds and their dependence on tropospheric cloud systems, J. Geophys. Res., 115, D00H18, doi:10.1029/2009JD012125, 2010.

Adriani, A., Massoli, P., Di Donfrancesco, G., Cairo, F., Moriconi, M. L., and Snels M.: Climatology of polar stratospheric clouds based on lidar observations from 1993 to 2001 over 
McMurdo Station, Antarctica, J. Geophys. Res., 109, D24211, doi:10.1029/2004JD004800, 2004.

Alexander, S. P., Klekociuk, A. R., Pitts, M. C., McDonald, A. J., and Arevalo-Torres, A.: The effect of orographic gravity waves on Antarctic polar stratospheric cloud occurrence and composition, J. Geophys. Res., 116, D06109, doi:10.1029/2010jd015184, 2011.

Butchart, N. and Remsberg, E. E.: The area of the stratospheric polar vortex as a diagnostic for tracer transport on an isentropic surface, J. Atmos. Sci., 43, 1319-1339, 1986.

Carslaw, K. S., Wirth, M., Tsias, A., Luo, B. P., Dörnbrack, A., Leutbecher, M., Volkert, H., Renger, W., Bacmeister, J. T., Reimer, E., and Peter, T.: Increased stratospheric ozone depletion due to mountain-induced atmospheric waves, Nature, 391, 675-678, doi:10.1038/35589, 1998.

Carslaw, K. S., Peter, T., Bacmeister, J. T., and Eckermann, S. D.: Widespread solid particle formation by mountain waves in the Arctic stratosphere, J. Geophys. Res., 104, 1827-1836, doi:10.1029/1998JD100033, 1999.

Chiou, E. W., McCormick, M. P., and Chu, W. P.: Global water vapor distributions in the stratosphere and upper troposphere derived from 5.5 years of SAGE II observations (1986-1991), J. Geophys. Res., 102, 19105-19118, 1997.

Dörnbrack, A. and Leutbecher, M.: Relevance of mountain waves for the formation of polar stratospheric clouds over Scandivania: A 20 year climatology, J. Geophys. Res., 106, 1583-1593, 2001.

Eckermann, S.D., Hoffmann, L., Höpfner, M., Wu, D. L., and Alexander, M. J.: Antarctic NAT PSC belt of June 2003: Observational validation of the mountain wave seeding hypothesis, Geophys. Res. Lett., 36, L02807, doi:10.1029/2008GL036629, 2009.

Fromm, M., Alfred, J., and Pitts, M.: A unified, long-term, highlatitude stratospheric aerosol and cloud database using SAM II, SAGE II, and POAM II/III data: Algorithm description, database definition, and climatology, J. Geophys. Res., 108, 4366, doi:10.1029/2002JD002772, 2003.

Fueglistaler, S., Luo, B. P., Voigt, C., Carslaw, K. S., and Peter, Th.: NAT-rock formation by mother clouds: a microphysical model study, Atmos. Chem. Phys., 2, 93-98, doi:10.5194/acp-293-2002, 2002.

Holton, J. R.: An introduction to Dynamic Meteorology, 4th ed., Academic Press, Burlington, USA, 535 pp., 2004.

Höpfner, M., Larsen, N., Spang, R., Luo, B. P., Ma, J., Svendsen, S. H., Eckermann, S. D., Knudsen, B., Massoli, P., Cairo, F., Stiller, G., v. Clarmann, T., and Fischer, H.: MIPAS detects Antarctic stratospheric belt of NAT PSCs caused by mountain waves, Atmos. Chem. Phys., 6, 1221-1230, doi:10.5194/acp-6-1221-2006, 2006.

Hoskins, B. J., McIntyre, M. E., and Robertson, A. W., On the use and significance of isentropic potential vorticity maps, Quart. J. Roy. Meteorol. Soc., 111, 877-946, doi:10.1002/qj.49711147002, 1985.

Jensen, E. J., Toon, O. B., Tabazadeh, A., and Drdla, K.: Impact of polar stratospheric cloud particle composition, number density, and lifetime on denitrification, J. Geophys. Res., 107, 8284, doi:10.1029/2001JD000440, 2002.

Kohma, M. and Sato, K.: The effects of atmospheric waves on the amounts of polar stratospheric clouds, Atmos. Chem. Phys., 11, 11535-11552, doi:10.5194/acp-11-11535-2011, 2011.
Kunz, A., Konopka, P., Muller, R., and Pan, L. L.: Dynamical tropopause based on isentropic potential vorticity gradients, J. Geophys. Res., 116, D01110, doi:10.1029/2010jd014343, 2011.

Lait, L. R.: An alternative form for potential vorticity, J. Atmos. Sci., 51, 1754-1759, 1994.

Lambert, A., Santee, M. L., Wu, D. L., and Chae, J. H.: A-train CALIOP and MLS observations of early winter Antarctic polar stratospheric clouds and nitric acid in 2008, Atmos. Chem. Phys., 12, 2899-2931, doi:10.5194/acp-12-2899-2012, 2012.

Larsen, N., Knudsen, B. M., Svendsen, S. H., Deshler, T., Rosen, J. M., Kivi, R., Weisser, C., Schreiner, J., Mauerberger, K., Cairo, F., Ovarlez, J., Oelhaf, H., and Spang, R.: Formation of solid particles in synoptic-scale Arctic PSCs in early winter 2002/2003, Atmos. Chem. Phys., 4, 2001-2013, doi:10.5194/acp-4-20012004, 2004.

Lowe, D. and MacKenzie, A. R.: Polar stratospheric cloud microphysics and chemistry, J. Atmos. Sol.-Terr. Phys., 70, 13-40, doi:10.1016/j.jastp.2007.09.011, 2008.

Nash, E. R., Newman, P. A., Rosenfield, J. E., and Schoeberl, M. R.: An objective determination of the polar vortex using Ertel's potential vorticity, J. Geophys. Res., 101, 9471-9478, doi:10.1029/96JD00066, 1996.

Palm, S. P., Fromm, M., and Spinhirne, J.: Observations of Antarctic polar stratospheric clouds by the Geoscience Laser Altimeter System (GLAS), Geophys. Res. Lett., 32, L22S04, doi:10.1029/2005g1023524, 2005.

Pan, L. L. and Munchak, L. A.: Relationship of cloud top to the tropopause and jet structure from CALIPSO data, J. Geophys. Res., 116, D12201, doi:10.1029/2010JD015462, 2011.

Pfister, L., Selkirk, H. B., Jensen, E. J., Podolske, J., Sachse, G., Avery, M., Schoeberl, M. R., Mahoney, M. J., and Richard, E.: Processes controlling water vapor in the winter Arctic tropopause region, J. Geophys. Res., 108, 8314, doi:10.1029/2001jd001067, 2002.

Pitts, M. C., Thomason, L. W., Poole, L. R., and Winker, D. M.: Characterization of Polar Stratospheric Clouds with spaceborne lidar: CALIPSO and the 2006 Antarctic season, Atmos. Chem. Phys., 7, 5207-5228, doi:10.5194/acp-7-5207-2007, 2007.

Pitts, M. C., Poole, L. R., and Thomason, L. W.: CALIPSO polar stratospheric cloud observations: second-generation detection algorithm and composition discrimination, Atmos. Chem. Phys., 9, 7577-7589, doi:10.5194/acp-9-7577-2009, 2009.

Poole, L. R. and Pitts, M. C.: Polar stratospheric cloud climatology based on stratospheric aerosol measurement II observations from 1978 to 1989, J. Geophys. Res., 99, 13083-13089, 1994.

Reichler, T., Dameris, M., and Sausen, R.: Determining the tropopause height from gridded data, Geophys. Res. Lett., 30, 2042, doi:10.1029/2003GL018240, 2003.

Riemann-Campe, K., Fraedrich, K., and Lunkeit, F.: Global climatology of convective available potential energy (CAPE) and convective inhibition (CIN) in ERA-40 reanalysis, Atmos. Res., 93, 534-545, 2009.

Schwierz, C., Croci-Maspoli, M., and Davies, H. C.: Perspicacious indicators of atmospheric blocking, Geophys. Res. Lett., 31, L06125, doi:10.1029/2003g1019341, 2004.

Solomon, S.: Stratospheric ozone depletion: A review of concepts and history, Rev. Geophys., 37, 275-316, 1999.

Teitelbaum, H., Moustaoui, M., and Fromm, M.: Exploring polar stratospheric cloud and ozone minihole formation: The primary 
importance of synoptic-scale flow perturbations, J. Geophys. Res., 106, 28173-28188, doi:10.1029/2000JD000065, 2001.

Vallis, G. K., Atmospheric and Oceanic Fluid Dynamics: Fundamentals and Large-Scale Circulation, Cambridge University Press, Cambridge, UK, 745 pp., 2006.

Wang, Z., Stephens, G., Deshler, T., Trepte, C., Parish, T., Vane, D., Winker, D., Liu, D., and Adhikari, L.: Association of Antarctic polar stratospheric cloud formation on tropospheric cloud systems, Geophys. Res. Lett., 35, L13806, doi:10.1029/2008gl034209, 2008.
Wilcox, L. J., Hoskins, B. J., and Shine, K. P.: A global blended tropopause based on ERA data. Part I: Climatology, Quart. J. Roy. Meteorol. Soc., 138, 561-575, doi:10.1002/qj.951, 2012.

Wirth, V.: Thermal versus dynamical tropopause in uppertropospheric balanced flow anomalies, Quart. J. Roy. Meteorol. Soc., 126, 299-317, doi:10.1002/qj.49712656215, 2000.

World Meteorological Organization (WMO): Definition of the tropopause, WMO Bull., 6, 136, 1957. 